\title{
Minimally-invasive-perventricular-device-occlusion versus surgical-closure for treating perimembranous-ventricular-septal- defect: 3-year outcomes of a multicenter randomized clinical trial
}

\author{
Wenxin Lu ${ }^{1 \#}$, Fengwen Zhang ${ }^{1 \#}$, Taibing Fan ${ }^{2}$, Tianli Zhao ${ }^{3}$, Yu Han ${ }^{2}$, Xiaopeng $\mathrm{Hu}^{1}$, Qi Li ${ }^{1}$, Hao Shi ${ }^{1}$, \\ Xiangbin Pan $^{1}$ \\ ${ }^{1}$ Structural Heart Disease Center, National Center for Cardiovascular Disease and Fuwai Hospital, Chinese Academy of Medical Sciences and \\ Peking Union Medical College, Beijing, China; ${ }^{2}$ Heart Center of Henan Provincial People's Hospital, Central China Fuwai Hospital of Zhengzhou \\ University, Zhengzhou, China; ${ }^{3}$ The Central South University, Changsha, China \\ Contributions: (I) Conception and design: X Pan, W Lu, F Zhang; (II) Administrative support: W Lu, F Zhang, T Fan, T Zhao, Y Han; (III) Provision \\ of study materials or patients: T Fan, T Zhao, Y Han, X Hu, Q Li, H Shi; (IV) Collection and assembly of data: W Lu, F Zhang, T Zhao, Y Han; (V) \\ Data analysis and interpretation: All authors; (VI) Manuscript writing: All authors; (VII) Final approval of manuscript: All authors. \\ "These authors contributed equally to this work. \\ Correspondence to: Xiangbin Pan. Structural Heart Disease Center, Fuwai Hospital, No. 167, North Lishi Street, Xicheng District, Beijing 100037, \\ China. Email: xiangbin428@hotmail.com.
}

Background: Minimally-invasive-perventricular-device-occlusion (MIPDO) combined superiority of surgical-repair and percutaneous-device-closure in treating perimembranous-ventricular-septal-defect (pmVSD). This study was to evaluate the efficacy and safety of MIPDO for treating pmVSD, comparing with surgical-repair.

Methods: Patients aged $\geq 3$ months with isolated pmVSDs were randomized to undergo either surgical or MIPDO procedure, with the median follow-up time of 49 months. The primary outcome was the rate of complete pmVSD closure at discharge. The secondary outcomes included the adverse events during hospitalization and follow-up, chest tube output volume, blood transfusion volume, procedural duration, ventilation time, hospitalization duration and hospitalization cost. Also, perioperative cardiac performance and systemic conditions were evaluated.

Results: Of the 313 patients ( 9 months to 42 years old; median, 4 years old) with pmVSDs recruited from 3 centers, 100 were finally enrolled and randomly allocated 1:1 into two groups. The non-inferiority (noninferiority margin $-8.0 \%$ ) of MIPDO to surgical closure regarding efficacy was shown in both intention-totreat (0, $95 \%$ CI: -0.055 to 0.055$)$ and per-protocol populations (0.02, 95\% CI: -0.018 to 0.058$)$. Although the rate of adverse events was similar between groups, the MIPDO group showed superiority in procedural duration, ventilation time, chest tube output volume, postoperative hospitalization time and hospitalization cost compared with surgical group $(\mathrm{P}<0.05)$. Moreover, MIPDO method showed comparable perioperative cardiac performance with milder change of systemic condition.

Conclusions: In patients with pmVSDs, MIPDO method showed non-inferiority to surgical closure in efficacy for both intention-to-treat and per-protocol population with easier perioperative recovery, economic benefit and promising outcomes.

Keywords: Perventricular; ventricular septal defect (VSD); minimally invasive; device; surgical

Submitted Nov 14, 2020. Accepted for publication Feb 06, 2021.

doi: $10.21037 /$ jtd-20-3298

View this article at: http://dx.doi.org/10.21037/jtd-20-3298 


\section{Introduction}

Ventricular septal defect (VSD) is one of the most common congenital cardiac malformations, among which perimembranous-ventricular-septal-defect (pmVSD) accounts for up to $80 \%$ (1). Surgical repair with cardiopulmonary bypass $(\mathrm{CPB})$ has been a routine treatment for decades, but the trauma and the potential risk of sequelae urge the innovation (2-5). In the last decade, with the development of devices, percutaneous closure gradually became an alternative for pmVSD treatment. However, due to its vascular approach, this method had some limitations in treating patients with low weight or dealing with tough cases, and complications (intraoperative aortic valve injury by wire, atrioventricular block or others) were frequently reported $(6,7)$. Thus, minimally-invasiveperventricular-device-occlusion (MIPDO), which combines the advantages of cardiac surgery and interventional technique, was proposed by surgeons (8-10). To place the device by a minimally invasive transthoracic procedure on a beating heart with the guidance of transoesophageal echocardiography (TEE) instead of the digital subtraction angiography, this operation is speculated to have easier postoperative recovery compared with surgical repair, as while as better controllability and less radiation exposure compared with percutaneous closure. Although MIPDO procedure has been widely applied in China and also adopted in some European countries (11-13), it is still controversial. We therefore performed this study to investigate the efficacy and safety of MIPDO procedure in treating patients with pmVSD. We present the following article in accordance with the CONSORT reporting checklist (available at http://dx.doi.org/10.21037/jtd-203298).

\section{Methods}

\section{Study design and patients}

This study was a prospective, multicenter, randomized controlled, open-label, non-inferiority trial evaluating the safety and efficacy of MIPDO method comparing to surgical closure for the treatment of isolated pmVSD patients. This study was registered with ClinicalTrials, Gov ID: NCT02644330. The study protocol was authorized by the Ethics Committee of Fuwai Hospital (Beijing, China) and conducted according to the principles expressed in the 1964 Declaration of Helsinki.

\section{Study methods}

The study was conducted in accordance with the Declaration of Helsinki (as revised in 2013). The study was approved by the Ethics Committee of Fuwai Hospital, Beijing, China (No. 2015-707) and informed consent was taken from all the patients. Patients were consecutively recruited from 3 centers during February 2016 to March 2017. Inclusion criteria for candidates were as follows: (I) age $\geq 3$ months; (II) transthoracic echocardiography (TTE)-confirmed isolated pmVSD, and diameter of defect ranged from 3 to $10 \mathrm{~mm}$ together with heart morphological changes; (III) applicability for both operation technique. Exclusion criteria included: (I) malaligned VSD; (II) muscular, subarterial or inlet septal VSD; (III) severe pulmonary artery hypertension with right-to-left shunt or bidirectional shunt; (IV) aortic valve prolapse with more than moderate regurgitation; (V) infective endocarditis, or with neoplasm in cardiac chambers; (VI) coexistence with other cardiac malformations that need surgery with CPB. All patients were routinely screened by clinical history collection, physical examination, chest X-rays, electrocardiography, TTE and blood tests. The patients were allocated 1:1 into two groups (MIPDO vs. surgical) in accordance to the randomized block design. The patients, surgeons and care givers were not blinded to the group assignment, while the assessing of outcomes was blinded. If the MIPDO strategy failed, patients would receive an intraoperative conversion to surgical closure.

\section{Operation technique and post-operation management}

For MIPDO group, procedures were executed in accordance with present clinical practice consensus (14) (Appendix 1). Perventricular closure was performed under TEE guidance with the ventricular occluder placed by a catheter directly entering the ventricle from a subxiphoid incision. Occluder size was similar to or slightly larger than the VSD (should not exceed $2 \mathrm{~mm}$ ). Concentric occluders were selected for patient with a margin of VSD $\geq 2 \mathrm{~mm}$ from the aortic annulus; and for those whose margins were less than $2 \mathrm{~mm}$, the eccentric occluders were recommended $(15,16)$. For surgical group, patients were operated under standard CPB through median sternotomy, and a dacron patch or direct suture was used to repair the pmVSD depending on its size. Operation rooms were equipped with both TEE and CPB machines, and surgeons qualified for both surgical and MIPDO methods were demanded in this 
trial.

Patients were given different postprocedural management between groups. Apart from the mutual intravenous antibiotics to prevent infection, patients in MIPDO group received postoperative heparin for 24 hours and oral aspirin $(3-5 \mathrm{mg} / \mathrm{kg} / \mathrm{d})$ for 6 months to prevent device-related thrombus, while those in surgical group were prescribed with oral digitalis and diuretic drugs for 3 months to improve cardiac function.

\section{Follow-up and evaluation criteria}

All patients underwent clinical examination, electrocardiography, chest X-rays, TTE at discharge, 1, 3, 6, 12, 24 and 36 months after operation. Besides, they receive daily blood tests during first 3 days after procedure.

The primary outcome was the success rate of the operation, which was defined as completing the certain procedure (consistent with the randomization when analyzed using intention-to-treat (ITT) population, while consistent with the ultimate effective protocol when analyzed using per-protocol (PP) population) with the TTE-confirmed complete closure of VSD at discharge. The secondary outcomes included the severe adverse events (SAEs) or adverse events (AEs) during hospitalization and followup, chest tube output volume, blood transfusion volume, operation time (from incision to complete closure of the sternum), ventilation time, postoperative hospitalization time and hospitalization cost. SAE was defined as one of the following events: all-cause death, cardiac-specific death, cardiac perforation, cardiac tamponade, iatrogenic tricuspid regurgitation, iatrogenic aortic regurgitation, dislocation or distortion of occluders and severe hemolysis. AE was defined as one of the following events: arrhythmia (complete atrioventricular block or complete bundle branch block), residual shunt (greater than $2 \mathrm{~mm}$ or flow rate greater than $3 \mathrm{~m} / \mathrm{s}$ ), infective endocarditis, undesirable healing or infection of the incision, hemorrhage and postoperative murmur.

Perioperative cardiac performance and systemic conditions were analyzed to delineate the immediate outcomes of different procedures. Perioperative cardiac performance was evaluated with TTE from baseline to 3-month post-operation (Appendix 2). Systemic conditions (including organ function, inflammatory response and hematological status) were assessed by the blood test results. The 3-day postprocedural examination results were used to illustrate patients' condition at discharge.

\section{Statistical analysis}

Sample size was determined according to the calculation result of sample size formula (Appendix 3), which indicated that a sample size of 50 participants for each group in a $1: 1$ ratio would provide more than $80 \%$ power in a 0.05 level test, with a one-sided alpha level of 0.025 , beta level of 0.2 and non-inferiority margin of $-0.08(-8 \%)$. Data was from the ITT (groups patients based on the randomization, irrespective of whether some of these patients might switch or discontinue treatment prior to the trial's end) and PP (groups patients based on the ultimate treatment) population. The primary outcomes were analyzed with data from both the ITT and PP population. The secondary outcomes were analyzed with data from the ITT population. Perioperative cardiac performance and systemic conditions were analyzed with data from the PP population. Statistical analysis was performed with SPSS 19.0 (IBM Corp, Armonk, New York). Continuous variables were analyzed using $t$ test (described with mean \pm standard deviation; independent-samples $t$ test used for data of different groups, paired-samples $t$ test used for data of different time points) or Mann-Whitney $U$ test (described with median and interquartile range) according to the distribution. Categorical variables (described with frequency and percentage) were analyzed using chi-square test or Fisher test according to the expected frequency. $\mathrm{P}$ values $<0.05$ were considered significant.

\section{Results}

Of the 100 patients consecutively included (ages ranged from 9 months to 42 years, median, 4 years; 47 males and 53 females), 50 patients were allocated to the MIPDO group and 50 patients to the surgical group, with the patients between 1 to 6-year-old dominating (74\%) (Table 1). The median duration of follow-up was 49 months (ranged from 43 to 56 months) and the completeness was $100 \%$. The flow of patient participation through this trial was shown in Figure 1.

The success rate of operation, set as the primary outcome to evaluate the efficacy, was $98 \%$ (49/50) in MIPDO group versus $98 \%$ (49/50) in surgical group for ITT population; and $100 \%$ (49/49) versus 98\% (50/51) for PP population. The absolute difference between groups for ITT population was 0 (95\% CI: -0.055 to 0.055 ); and for PP population 
Table 1 Demographic and VSD characters between groups

\begin{tabular}{|c|c|c|}
\hline & MIPDO $(\mathrm{N}=50)$ & Surgical $(N=50)$ \\
\hline Age (year) & 4 (2 to 5 ) & 4 (2 to 5 ) \\
\hline$<1$ year $(n)$ & 1 & 8 \\
\hline $1-6$ year $(n)$ & 42 & 32 \\
\hline$>18$ year $(n)$ & 3 & 4 \\
\hline Height (cm) & 91.5 (79.8 to 103.0$)$ & 90.5 (77.3 to 107.3$)$ \\
\hline Weight (kg) & 13 (10.3 to 16.1$)$ & 13.0 (8.5 to 17$)$ \\
\hline Size of VSD (mm) & 5 (4 to 6$)$ & $6(5$ to 7$)$ \\
\hline
\end{tabular}

VSD, ventricular septal defect.

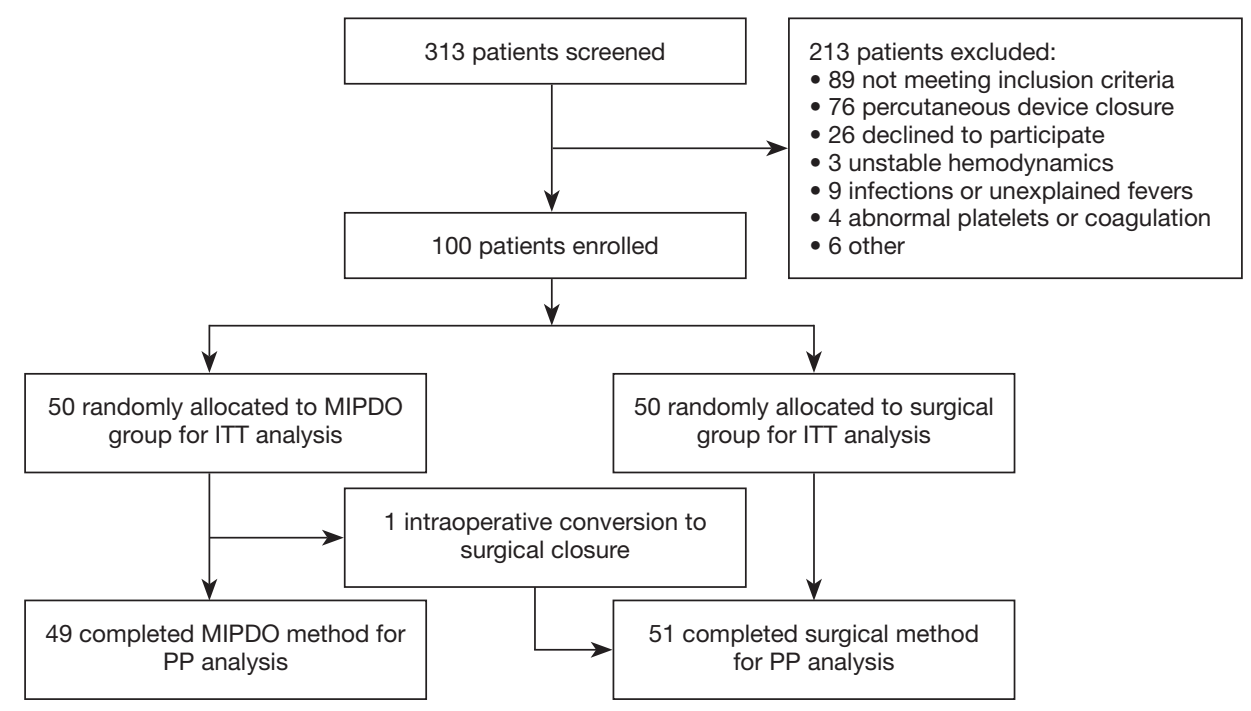

Figure 1 Study design and patient participation flow. ITT, intention-to-treat; PP, per-protocol.

was 0.02 (95\% CI: -0.018 to 0.058 ). For both populations, the lower limit of the $95 \% \mathrm{CI}$ : was beyond the predefined non-inferiority margin of -0.08 , suggesting the noninferiority of MIPDO method compared with surgical closure in treating pmVSD (Table 2). Here were the details of 2 failed cases: case 1 was an intraoperative conversion to surgical closure, the patient had a $7.5 \mathrm{~mm} \mathrm{VSD}$ and the distance from aortic annulus to VSD margin was $1.8 \mathrm{~mm}$, with no residual shunt at discharge; case 2 was randomized to surgical group, showing a residual shunt of $1 \mathrm{~mm}$ at discharge, which disappeared at 1-month follow-up.

In MIPDO group, AE was found in one patient who had ECG-confirmed postoperative arrhythmia (left anterior fascicular block) at 1 - and 3-month follow-up, whereas recovered at 6-month post-procedural examination. In surgical group, AEs were revealed in 2 cases. One was for undesirable incision healing with granulomas formation, and underwent debridement at 1 month after surgery. The other was for incision inflammation, which was presented 2 weeks after surgery, and recovered with intravenous 
Table 2 Efficacy endpoint between procedures in different patterns of population

\begin{tabular}{lccc}
\hline & MIPDO $(\mathrm{n} / \mathrm{N})$ & Surgical $(\mathrm{n} / \mathrm{N})$ & Difference between groups (two-sided 95\% Cl) \\
\hline ITT population & $49 / 50(98 \%)$ & $49 / 50(98 \%)$ & $0(-0.055 \text { to } 0.055)^{\star}$ \\
PP population & $49 / 49(100 \%)$ & $50 / 51(98 \%)$ & $0.02(-0.018 \text { to } 0.058)^{\star}$ \\
\hline
\end{tabular}

*, showing non-inferiority of perventricular to surgical closure. $\mathrm{n}$, population with echocardiography-confirmed complete VSD closure; N, population in the different patterns of the predefined population; ITT, intention-to-treat; PP, per-protocol.

Table 3 Reported adverse events between procedures in intention-to-treat population

\begin{tabular}{|c|c|c|c|}
\hline & MIPDO $(\mathrm{N}=50)$ & Surgical $(N=50)$ & Difference between groups $(95 \% \mathrm{Cl})$ \\
\hline All-cause death & 0 & 0 & - \\
\hline Cardiac-specific death & 0 & 0 & - \\
\hline Cardiac perforation or tamponade & 0 & 0 & - \\
\hline Dislocation or distortion of occluders & 0 & 0 & - \\
\hline Severe hemolysis & 0 & 0 & - \\
\hline Adverse events (n) & 1 & 2 & $-0.02(-0.087$ to 0.047$)$ \\
\hline Arrhythmia & $1^{\mathrm{a}}$ & 0 & $0.02(-0.019$ to 0.059$)$ \\
\hline Infective endocarditis & 0 & 0 & - \\
\hline Hemorrhage & 0 & 0 & - \\
\hline Postoperative murmur & 0 & 0 & - \\
\hline
\end{tabular}

a, newly onset of 'left anterior fascicular block', recovered at 6-month post-procedural examination.

Table 4 Safety and economical endpoints between procedures in intention-to-treat population

\begin{tabular}{|c|c|c|c|}
\hline & MIPDO $(\mathrm{N}=50)$ & Surgical repair $(\mathrm{N}=50)$ & $P$ value \\
\hline Ventilation time $(\mathrm{h})$ & $4.7 \pm 2.1$ & $9.8 \pm 12.6$ & 0.005 \\
\hline Chest tube output (mL) & $77.5 \pm 74.0$ & $130.6 \pm 124.5$ & 0.01 \\
\hline Size of occluder (mm) & $6.1 \pm 1.4$ & - & - \\
\hline Hospitalization cost $(¥)$ & $38,142.9(35,616.7$ to $42,750.0)$ & $44,383.8(39,911.5$ to $48,275.0)$ & $<0.001$ \\
\hline
\end{tabular}

antibiotics used. No other complications or reintervention occurred throughout the follow-up (Table 3). Although the efficacy endpoint and complication rate of the two groups were similar, the MIPDO group showed significantly lower operation time, ventilation time, chest tube output volume, postoperative hospitalization time and hospitalization cost than that in surgical group for the ITT population $(\mathrm{P}<0.05$, Table 4).

Furthermore, perioperative cardiac performance and systemic condition were analyzed in PP population. 
Table 5 Peri-operational cardiac performance between procedures in per-protocol population

\begin{tabular}{|c|c|c|c|c|c|c|c|c|}
\hline & \multicolumn{4}{|c|}{ MIPDO (N=49) } & \multicolumn{4}{|c|}{ Surgical $(\mathrm{N}=51)$} \\
\hline \multicolumn{9}{|c|}{ Structural remodeling } \\
\hline $\mathrm{LA}(\mathrm{mm})$ & 21 (20 to 22 ) & $19(17 \text { to } 21)^{\ddagger}$ & $19(17 \text { to } 21)^{\ddagger}$ & $20(18 \text { to } 20)^{\dagger}$ & 22 (19 to 24$)$ & $20(17 \text { to } 22)^{\ddagger}$ & $19(18 \text { to } 22)^{\ddagger}$ & $19(17 \text { to } 22)^{\ddagger}$ \\
\hline LVEDd (mm) & 33 (30 to 36$)$ & $28(25 \text { to } 31)^{\ddagger}$ & $28(26 \text { to } 32)^{\ddagger}$ & $29(26 \text { to } 31)^{\ddagger}$ & 34 (30 to 37$)$ & $28(25 \text { to } 32)^{\ddagger}$ & $29(26 \text { to } 32)^{\ddagger}$ & $29(26 \text { to } 32)^{\ddagger}$ \\
\hline IVS/LVPW & 1 (1 to 1$)$ & 1 (1 to 1$)$ & 1 (1 to 1$)$ & 1 (1 to 1$)$ & 1 (1 to 1$)$ & $1(1$ to 1$)$ & $1(1$ to 1$)$ & $1(1$ to 1$)$ \\
\hline \multicolumn{9}{|c|}{ Myocardial function } \\
\hline $\mathrm{EF}(\%)$ & 67 (65 to 70$)$ & 66 (60 to 70$)$ & 67 (65 to 70$)$ & $67(65 \text { to } 70)^{\pi}$ & 65 (63 to 69$)$ & 65 (62 to 66$)$ & 65 (63 to 68$)$ & $68(65 \text { to } 70)^{\pi}$ \\
\hline \multicolumn{9}{|c|}{ Hemodynamic status } \\
\hline
\end{tabular}

${ }^{a}$, measured at 3-day after procedure. ${ }^{\dagger} \mathrm{P}<0.05$, ${ }^{\ddagger} \mathrm{P}<0.001$ as compared with baseline; ${ }^{9} \mathrm{P}<0.001$ as compared with discharge. LA, left atrial diameter; LVEDd, left ventricular end-diastolic dimension; RV, right ventricular diameter; IVS, interventricular septal thickness; LVPW, left ventricular posterior wall thickness; $\mathrm{EF}$, ejection fraction; $\mathrm{HR}$, heart rate; $\mathrm{Cl}$, cardiac index.

Perioperative TTE showed similar trend of cardiac performance in two groups (Table 5). Perioperative blood test results indicated that creatine kinase (CK) and lactate dehydrogenase (LDH) levels were significantly increased postoperatively, as while as hemoglobin $(\mathrm{Hb})$, platelet count (PLT), alanine transaminase (ALT) and creatine kinase-MB (CK-MB) levels were significantly decreased in both groups. White blood cell count (WBC) and blood urea nitrogen (BUN) levels were significantly increased only in surgical group, while the aspartate transaminase (AST) level was significantly decreased only in MIPDO group. Additionally, the MIPDO group showed significantly less variation of WBC, PLT, CK, LDH and BUN, and greater amelioration of AST compared with surgical group $(\mathrm{P}<0.05$, Table 6).

\section{Discussion}

To our best knowledge, this is the first study demonstrating the non-inferiority of MIPDO to surgical closure in efficacy for both ITT and PP population with a median followup time of 49 months. In this study, data were analyzed with 'ITT' or 'PP' population for different practical concerns: the 'ITT' emphasized the 'intention', better for evaluating the rationality of clinical decision making; while 'PP' emphasized the 'protocol', better for evaluating the technique itself. The rate of effective VSD closure with
MIPDO method (98\%) in ITT population was comparable to previous reports (17-19), and the complication rate (2\%) was low throughout the follow-up. Moreover, MIPDO showed superiority in operation-related trauma (less operation time), postoperative recovery (less ventilation time, chest tube output and postoperative hospitalization time) and economic benefit (lower hospitalization cost).

Although MIPDO method has been popular for its short learning curve, easy manipulation, satisfying short-term and long-term outcomes in China since firstly proposed around 2008 by Zeng and colleagues $(16,20)$, it has still not been extensively adopted by the international community. The real potential advantages of this technique remain ambiguous and the patient-selection indications have not reached an agreement in different centers, which seems to be the core issues for the development and promotion of this procedure.

In terms of patient-selection indications, babies (usually less than 1-year-old) with pmVSDs suitable for device closure were previously supposed as the only beneficiaries of this technique, but actually, some other patients could also take benefit from MIPDO. In our center, not like completely percutaneous closure, candidates for MIPDO procedure had following characteristics: (I) small age and low weight: which closely related to poor vascular condition and higher rate of vascular complications, empirically, we 
Table 6 Peri-operational systemic condition between procedures in per-protocol population

\begin{tabular}{|c|c|c|c|c|c|c|c|}
\hline & \multicolumn{3}{|c|}{ MIPDO $(\mathrm{N}=49)$} & \multicolumn{3}{|c|}{ Surgical $(\mathrm{N}=51)$} & $P$ value \\
\hline WBC $\left(10^{9} / \mathrm{L}\right)$ & $8.06 \pm 2.35$ & $8.50 \pm 2.83$ & 0.26 & $8.92 \pm 2.71$ & $10.86 \pm 3.39$ & $<0.001$ & $<0.001$ \\
\hline $\mathrm{Hb}(\mathrm{g} / \mathrm{L})$ & $119.46 \pm 12.23$ & $108.94 \pm 13.49$ & $<0.001$ & $120.54 \pm 16.23$ & $105.58 \pm 13.87$ & $<0.001$ & 0.22 \\
\hline PLT $\left(10^{9} / \mathrm{L}\right)$ & 299 (260 to 331 ) & 249 (188 to 313$)$ & $<0.001$ & 302 (255 to 366$)$ & 210 (167 to 238$)$ & $<0.001$ & 0.002 \\
\hline AST (IU/L) & 31 (27 to 38 ) & 28 (22 to 34$)$ & 0.001 & 32 (26 to 38$)$ & 32 (27 to 42 ) & 0.59 & 0.002 \\
\hline CK-MB (IU/L) & 21 (4 to 29 ) & $13(10$ to 15$)$ & $<0.001$ & 20 (6 to 25$)$ & $12(10$ to 16$)$ & $<0.001$ & 0.23 \\
\hline CK (IU/L) & 74 (49 to 102$)$ & 107 (66 to 160$)$ & $<0.01$ & 91 (62 to 120$)$ & 267 (145 to 407$)$ & $<0.001$ & $<0.001$ \\
\hline LDH (IU/L) & 240 (96 to 270$)$ & 242 (169 to 313$)$ & 0.001 & 219 (124 to 264$)$ & 340 (282 to 408$)$ & $<0.001$ & $<0.001$ \\
\hline
\end{tabular}

WBC, white blood cell count; Hb, hemoglobin; PLT, platelet count; ALT, alanine transaminase; AST, aspartate transaminase; CK-MB, creatine kinase-MB; CK, creatine kinase; LDH, lactate dehydrogenase; Scr, serum creatinine; BUN, blood urea nitrogen. ${ }^{a}$ comparison of variation from baseline to discharge between groups; ${ }^{b}$ measured at 3-day after procedure.

use ' 1 year' and ' $10 \mathrm{~kg}$ ' as the thresholds; (II) VSD located close to the aortic valve: compared with percutaneous method, the direct vision and easier manipulation of MIPDO method could help to locate the device more precisely, which to some extent might decrease the device/ operation-related complications (including valve injury and arrhythmia), empirically, we use the distance of ' $2 \mathrm{~mm}$ ' as the threshold (21-23); (III) patients with contraindications to medical radiation or contrast agents: attributed to the intraoperative TEE guidance, good controllability could be achieved in MIPDO method, which might be the gospel for such patients; (IV) willingness of patients or their legal guardians: last but not least, some patients (or their legal guardians) in China are prudent and conservative, they tend to choose a procedure 'preparing for more eventualities'. In current study, patients were enrolled with a wide range of ages and had both restrictive and nonrestrictive pmVSDs. Actually, we performed MIPDO closure for 9 patients less than 1-year-old, 27 patients with small distance between VSD and aortic valve $(<2 \mathrm{~mm})$ and 2 patients with contraindications to the contrast agents. Valve disfunction and arrhythmias (complete atrioventricular block was the most serious while right bundle branch block was the most common) were usually reported as main complications associated with percutaneous VSD closure (24-26). Nevertheless, in this study, no valve disfunction was revealed and only 1 case of left anterior fascicular block (reverted to normal within 6-month after operation) was reported in patients undergoing MIPDO procedure.

On the other hand, the MIPDO procedure shows superiority to the surgery in terms of minimal invasion. Although without extra oral digitalis and diuretic drugs for 3 months like the surgical group, the MIPDO group showed comparable perioperative cardiac performance including the postoperative diminution of left atrial diameter, left ventricular end-diastolic dimension and right ventricular diameter, these immediate outcomes of MIPDO were consistent with the expected hemodynamic change: once the VSD was closed, the overloaded right ventricle and left atrium would tent to become normalized and initiate structural remodeling. Furthermore, patients in the MIPDO group showed minor elevation of WBC, $\mathrm{CK}, \mathrm{LDH}$ and BUN, outgrowth of slighter inflammatory response (27) and milder renal function impairment $(28,29)$. Although patients in MIPDO group were prescribed with heparin and oral aspirin, they presented similar or even less variation in PLT compared with surgical groups.

What should be mentioned was that the continuous downtrend of cardiac index (CI) was observed in both groups during the first 3-month follow-up. Although the declination of CI was frequently attributed to the perioperative impairment of the myocardial function (30), we speculated that the elevated efficiency of the heart pump might be also a reasonable explanation, and the 
TTE at both resting and with workload might provide more comprehensive information. Another unexpected phenomenon was the paradoxical post-procedural declination of enzymes (ALT, CK-MB in both groups and AST in MIPDO group), which might be attributed to the perioperative application of cardiotrophin and liverprotecting drugs.

Potential limitations of present trial emerged during result interpretation. Firstly, we speculated that evaluation of cardiac performance with workload might be important supplement to present study, since the VSD closure and the perioperative stress might cast contradictory influence toward the hemodynamic status. Secondly, perioperative management was different between groups in accordance with the routine of participating centers, although designed for better reflection of the real world and avoidance of unnecessary adverse drug reactions (such as electrolyte imbalance or conduction block), it might induce some bias. Also, the larger sample size and longer-term follow-up are needed for more robust evidence.

\section{Conclusions}

In patients with pmVSDs, MIPDO method showed noninferiority to surgical closure in efficacy for both ITT and PP population with easier perioperative recovery, economic benefit and promising outcomes.

\section{Acknowledgments}

Funding: This work was supported by the National Key Research and Development Program of China (2016YFC1302004), National Natural Science Foundation of China (81670289), CAMS Innovation Fund for Medical Sciences (2017-I2M-4-001) and Medical Science and Technology Project of Henan Province and Ministry Coconstruction (SB201902031).

\section{Footnote}

Reporting Checklist: The authors have completed the CONSORT reporting checklist. Available at http://dx.doi. org/10.21037/jtd-20-3298

Data Sharing Statement: Available at http://dx.doi. org/10.21037/jtd-20-3298

Conflicts of Interest: All authors have completed the ICMJE uniform disclosure form (available at http://dx.doi. org/10.21037/jtd-20-3298). The authors have no conflicts of interest to declare.

Ethical Statement: The authors are accountable for all aspects of the work in ensuring that questions related to the accuracy or integrity of any part of the work are appropriately investigated and resolved. The study was conducted in accordance with the Declaration of Helsinki (as revised in 2013). The study was approved by the Ethics Committee of Fuwai Hospital, Beijing, China (No. 2015707 ) and informed consent was taken from all the patients.

Open Access Statement: This is an Open Access article distributed in accordance with the Creative Commons Attribution-NonCommercial-NoDerivs 4.0 International License (CC BY-NC-ND 4.0), which permits the noncommercial replication and distribution of the article with the strict proviso that no changes or edits are made and the original work is properly cited (including links to both the formal publication through the relevant DOI and the license). See: https://creativecommons.org/licenses/by-nc-nd/4.0/.

\section{References}

1. Roos-Hesselink JW, Meijboom FJ, Spitaels SE, et al. The outcome of patients after surgical closure of ventricular septal defect at a young age: longitudinal follow-up of 2234 years. Eur Heart J 2004;25:1057-62.

2. Zhao LJ, Han B, Zhang JJ, et al. Postprocedural outcomes and risk factors for arrhythmias following transcatheter closure of congenital perimembranous ventricular septal defect: a single-center retrospective study. Chin Med J (Engl) 2017;130:516-21.

3. Schipper M, Slieker MG, Schoof PH, et al. Surgical repair of ventricular septal defect; contemporary results and risk factors for a complicated course. Pediatr Cardiol 2017;38:264-70.

4. Omelchenko AY, Zhuang Z, Schreiber C, et al. Surgical off-pump closure of perimembranous ventricular septal defects. Asian Cardiovasc Thorac Ann 2014;22:31-5.

5. Heiberg J, Ringgaard S, Schmidt MR, et al. Structural and functional alterations of the right ventricle are common in adults operated for ventricular septal defect as toddlers. Eur Heart J Cardiovasc Imaging 2015;16:483-9.

6. Holzer R, de Giovanni J, Walsh KP, et al. Transcatheter closure of perimembranous ventricular septal defects using the Amplatzer membranous VSD occluder: immediate 
and midterm results of an international registry. Catheter Cardiovasc Interv 2006;68:620-8.

7. Fu YC, Bass J, Amin Z, et al. Transcatheter closure of perimembranous ventricular septal defects using the new Amplatzer membranous VSD occluder: results of the U.S. phase I trial. J Am Coll Cardiol 2006;47:319-25.

8. Zeng XJ, Sun SQ, Chen XF, et al. Device closure of perimembranous ventricular septal defects with a minimally invasive technique in 12 patients. Ann Thorac Surg 2008;85:192-4.

9. Xing Q, Wu Q, Shi L, et al. Minimally invasive transthoracic device closure of isolated ventricular septal defects without cardiopulmonary bypass: longterm follow-up results. J Thorac Cardiovasc Surg 2015;149:257-64.

10. Ou-Yang WB, Li SJ, Wang SZ, et al. Echocardiographic guided closure of perimembranous ventricular septal defects. Ann Thorac Surg 2015;100:1398-402.

11. Schreiber C, Vogt M, Kühn A, et al. Perventricular closure of a perimembranous VSD: treatment option in selected patients. Thorac Cardiovasc Surg 2012;60:78-80.

12. Aboulhosn J, Levi D, Sopher M, et al. Perventricular closure of a large ventricular septal defect in congenitally corrected transposition of the great arteries. Congenit Heart Dis 2010;5:60-5.

13. Michel-Behnke I, Ewert P, Koch A, et al. Device closure of ventricular septal defects by hybrid procedures: a multicenter retrospective study. Catheter Cardiovasc Interv 2011;77:242-51.

14. Chinese Association of Cardiovascular Surgeons. Chinese expert consensus statement on minimally invasive transthoracic device closure of ventricular septal defects. Chin J Thorac Cardiovasc Surg 2011;27:516-8.

15. Zhang GC, Chen Q, Cao H, et al. Minimally invasive perventricular device closure of ventricular septal defect in infants under transthoracic echocardiograhic guidance: feasibility and comparison with transesophageal echocardiography. Cardiovasc Ultrasound 2013;11:8.

16. Xing Q, Pan S, An Q, et al. Minimally invasive perventricular device closure of perimembranous ventricular septal defect without cardiopulmonary bypass: multicenter experience and mid-term follow-up. J Thorac Cardiovasc Surg 2010;139:1409-15.

17. Fang GH, Chen Q, Hong ZN, et al. The comparison of perventricular device closure with transcatheter device closure and the surgical repair via median Sternotomy for perimembranous ventricular septal defect. Ann Thorac Cardiovasc Surg 2018;24:308-14.
18. Huang JS, Huang ST, Sun KP, et al. Health-related quality of life in children and adolescents undergoing intraoperative device closure of isolated perimembranous ventricular septal defects in southeastern China. J Cardiothorac Surg 2019;14:218-23.

19. Chen Q, Hong ZN, Zhang GC, et al. Intraoperative device closure of isolated ventricular Septal defects: experience on 1,090 cases. Ann Thorac Surg 2018;105:1797-802.

20. Sun Y, Zhu P, Zhou PY, et al. Intra-operative device closure of perimembranous ventricular septal defect without cardiopulmonary bypass under guidance of transepicardial echocardiography: a single center experience. J Cardiothorac Surg 2016;11:87.

21. Congenital Heart Disease Committee, Chinese Pediatric Physicians Association, Chinese Medical Doctor Association; Subspecialty Group of Cardiology, the Society of Pediatrics, Chinese Medical Association; Editorial Board, Chinese Journal of Pediatrics. Expert consensus for the interventional treatment of pediatric congenital heart disease. Chin J Pediatr 2015;53:17-24.

22. Walavalkar V, Maiya S, Pujar S, et al. Percutaneous device closure of congenital isolated ventricular septal defects: a single-center retrospective database study amongst 412 cases. Pediatr Cardiol 2020;41:591-8.

23. Expert working group on interventional diagnosis and treatment of cardiovascular diseases through surgical approaches, National health commission of the People's Republic of China. Expert consensus for the interventional diagnosis and treatment of common cardiovascular diseases through surgical approaches. Chinese Circulation Journal 2017;32:105-19.

24. Odemis E, Saygi M, Guzeltas A, et al. Transcatheter closure of perimembranous ventricular septal defects using Nit-Occlud((R)) Le VSD coil: early and mid-term results. Pediatric cardiology 2014;35:817-23.

25. Landman G, Kipps A, Moore P, et al. Outcomes of a modified approach to transcatheter closure of perimembranous ventricular septal defects. Catheter Cardiovasc Interv 2013;82:143-9.

26. Wu H, Qin Y, Zhao X, et al. Transcatheter closure of multi-hole perimembranous VSD with aneurysm: 3-year follow-up study. Clin Res Cardiol 2009;98:563-9.

27. Lundin ES, Wodlin NB, Nilsson L, et al. Markers of tissue damage and inflammation after robotic and abdominal hysterectomy in early endometrial cancer: a randomised controlled trial. Sci Rep 2020;10:7226.

28. Magden K, Yildirim I, Kutu M, et al. Recovery process in patients followed-up due to acute kidney injury. 
Hippokratia 2013;17:239-42.

29. Gao J, Chen M, Wang X, et al. Risk factors and prognosis of acute kidney injury in adult hospitalized patients: a twoyear outcome. Minerva Urol Nefrol 2015;67:179-85.

Cite this article as: Lu W, Zhang F, Fan T, Zhao T, Han Y, Hu X, Li Q, Shi H, Pan X. Minimally-invasive-perventriculardevice-occlusion versus surgical-closure for treating perimembranous-ventricular-septal-defect: 3-year outcomes of a multicenter randomized clinical trial. J Thorac Dis 2021;13(4):2106-2115. doi: 10.21037/jtd-20-3298
30. Liu H, Lu FX, Zhou J, et al. Minimally invasive perventricular versus open surgical ventricular septal defect closure in infants and children: a randomised clinical trial. Heart 2018;104:2035-43. 


\section{Appendix 1: details of MIPDO and surgical procedures}

\section{MIPDO procedure}

Patients had a preoperative fasting and water time of 5 to 6 hours and received general anesthesia with endotracheal intubation in a standard operating room. The TEE probe was inserted, and the procedure was performed under TEE guidance. The location, size, flow direction of the pmVSD, valvular regurgitation, and distance between the pmVSD and the aortic valve were measured intraoperatively by TEE. Antibiotics and heparin $(80 \mathrm{IU} / \mathrm{kg}$ ) were administered intravenously before the operation. A median thoracic skin incision of 1 to $2 \mathrm{~cm}$ was made. Then, the subcutaneous tissue was dissected to the left fourth parasternal intercostal space. The intercostal muscles were dissected to establish the surgical approach. The free wall of the right ventricle was exposed by opening and suspending the pericardium. A purse-string suture was placed on the right ventricular free wall directly facing the direction of the pmVSD shunt. The right ventricle was punctured within the purse-string suture with a trocar. A 0.035 -inch guide wire was placed in the trocar. After the guide wire was passed through the pmVSD, the trocar was removed and the dilator and delivery sheath were advanced through the pmVSD to the left ventricle along the guide wire. The size of the occluder was 1 to $2 \mathrm{~mm}$ larger than the diameter of the pmVSD. After removing the guide wire and dilator sheath, the selected occluder was deployed through the delivery sheath under the guidance of TEE. The TEE was used to reassess the shape and position of the occluder, the presence of a residual shunt, and valvular regurgitation before and after occluder release. Finally, the delivery sheath was withdrawn, and the purse-string suture was tied using the knotter. After completing the operation, the patient was sent to the intensive care unit where the endotracheal tube was removed. Echocardiography and electrocardiogram were performed to confirm that there was no obvious RS or server arrhythmia after the operation right in the operation room.

\section{Devices and delivery system:}

The symmetric occluders (used for pmVSDs with a distance of more than $2 \mathrm{~mm}$ from the aortic valve) were of following manufacturers: (I) Shanghai Shape Memory Alloy Co, Ltd, Huangpu, Shanghai, China; (II) Lifetech scientific Co, Ltd, Shenzhen, China; (III) Beijing Huayi Shengjie Science\&Technology Co, Ltd, Beijing, China. The asymmetric occluders (used for pmVSDs with a distance of less than $2 \mathrm{~mm}$ from the aortic valve) were all from Shanghai Shape Memory Alloy Co, Ltd, Huangpu, Shanghai, China.

The entire delivery system includes a trocar, 0.035 -inch guide wire, dilator and delivery sheath, and a loading sheath. The size of the delivery sheath ( 5 to $9 \mathrm{Fr}$ ) is chosen according to the size of the occluder. Thoracoscopic instruments, including a retractor and a knotter, were used to perform the procedures through a 1 to $2 \mathrm{~cm}$ surgical incision.

\section{Surgical procedure}

Patients had a preoperative fasting and water time of 5 to 6 hours and received general anesthesia with endotracheal intubation in a standard operating room. A mid-line sternotomy incision thoracotomy of 10 to $20 \mathrm{~cm}$ in length was made by using moderate hypothermia at 32-34, CPB, aortic cross-clamping and cold crystalloid cardioplegic arrest. The defect was reached using an incision in the right atrium. Intraoperative temporary detachment of the tricuspid valve increased the exposure of the defect. Depending on the size of the defect and surgeon preference, the VSD was closed by direct suture or with a Dacron patch using an interrupted suture. Careful examination was needed to confirm that there was no obvious residual shunt, tricuspid valve regurgitation or server arrhythmia after the heart resuscitation. Protamine was given intravenously $(2.0 \mathrm{mg} / \mathrm{kg})$ following the termination of CPB. 

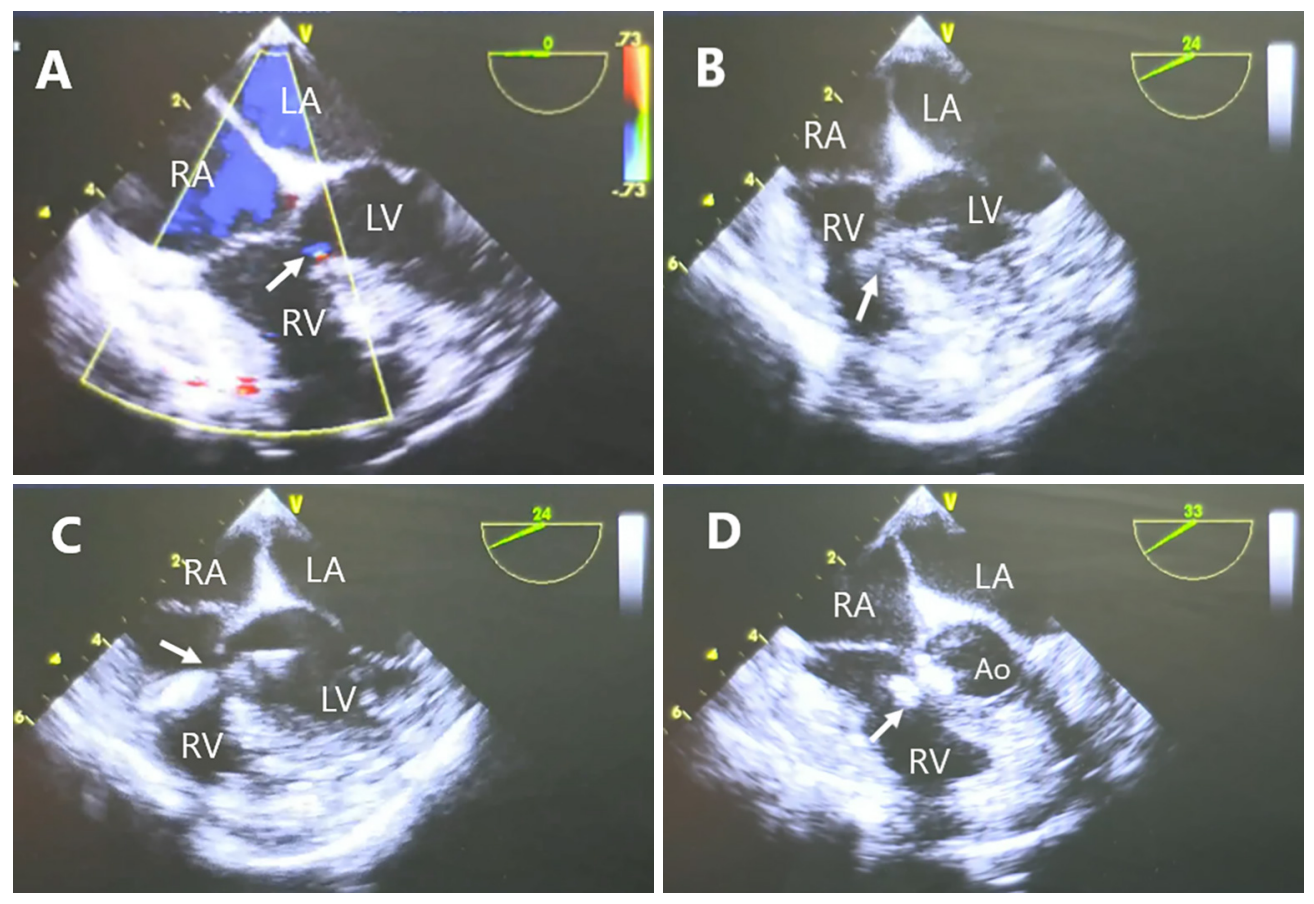

Figure S1 Transesophageal echocardiogram views of the MIPDO procedure. (A) Perimembranous ventricular septal defects (pmVSDs) before MIPDO procedure (arrow). (B) The guide wire (arrow) was inserted through the pmVSD to the left ventricle (LV). (C) The delivery sheath (arrow) was inserted through the pmVSD. (D) Occluder (arrow) and aortic valve under the long axis view of the aorta. Ao, aorta; LA, left atrium; LV, left ventricle; RA, right atrium; RV, right ventricle. 


\section{Appendix 2: measurement and calculation formulas of the echo data}

Parameters including LA, RV, IVS, LVPW, EF, MDG, TDG, ASG, PSG were measured directly with the echocardiography. The HR was measured with the simultaneous electrocardiography. CI was calculated using original indexes of weight, LVEDD and HR with the following formulas:

$\mathrm{CI}\left[\mathrm{L} /\left(\mathrm{min} \cdot \mathrm{m}^{2}\right)\right]=\operatorname{LVEDV}(\mathrm{mL}) * \mathrm{EF}(\%) * \mathrm{HR}(\mathrm{bpm}) /\left(\mathrm{BSA}\left(\mathrm{m}^{2}\right)^{*} 100000\right)$;

$\operatorname{LVEDV}(\mathrm{ml})=7$ * $\operatorname{LVEDD}(\mathrm{cm})^{3} /(2.4+\operatorname{LVEDD}(\mathrm{cm}))$;

BSA $\left(\mathrm{m}^{2}\right)=$ weight $(\mathrm{kg})^{*} 0.035+0.1$, when weight $<30 \mathrm{~kg}$;

BSA $\left(\mathrm{m}^{2}\right)=(\text { weight }(\mathrm{kg})-30)^{*} 0.02+1.05$, when weight $\geq 30 \mathrm{~kg}$. 


\section{Appendix 3: calculation of sample size}

We used the sample size formula as follows:

$$
n=\frac{\left[\mu_{1-\alpha} \sqrt{2 \bar{p}(1-\bar{p})}+\mu_{1-\beta} \sqrt{p_{T}\left(1-p_{T}\right)+p_{C}\left(1-p_{C}\right)}\right]^{2}}{\left(\Delta-\left(p_{T}-p_{C}\right)\right)^{2}}
$$

Definitions and values used (decided according to the clinical consensus and articles reviewed) in the formula:

$p_{T}$ :estimated successful rate of MIPDO group (98\%); $p_{C}$ : estimated successful rate of surgical group (98\%); $\bar{p}=\left(p_{T}+p_{C}\right) / 2$; $\Delta$ : non-inferiority margin (8\%); $\mu$ : The quantile corresponding to a standard normal distribution, $\alpha$ : Type I error levels for statistical tests ( 0.025 one-sided); $\beta$ : Type II error levels for statistical tests ( 0.2 one-sided). 